\title{
Affect, Learning, and Delight
}

\author{
James C. Lester \\ Department of Computer Science, North Carolina State University, \\ Raleigh, North Carolina, USA 27695 \\ lester@ncsu.edu
}

\begin{abstract}
Because of the growing recognition of the role that affect plays in learning, affective computing has become the subject of increasing attention in research on interactive learning environments. The intelligent tutoring systems community has begun actively exploring computational models of affect, and game-based learning environments present a significant opportunity for investigating student affect in interactive learning. One family of game-based learning environments, narrative-centered learning environments, offer a particularly compelling laboratory for investigating student affect. In narrativecentered environments, learning activities play out in dynamically generated interactive narratives and training scenarios. These afford significant opportunities for investigating computational models of student emotion. In this talk, we explore the role that affective computing can play in next-generation interactive learning environments, with a particular focus on affect recognition, affect understanding, and affect synthesis in game-based learning.
\end{abstract}

Keywords: Affective computing, intelligent tutoring systems, game-based learning. 